\title{
Midwives' experiences of caring for women with antenatal anxiety and depression: A qualitative study with midwifery acupuncturists
}

Lee-Ana Lowe ${ }^{A, B}$ MHS (Chinese Medicine), BHS (Acupuncture) - Debra Betts ${ }^{C}$ PhD, BHS (Acupunture)

${ }^{\mathrm{A}}$ Corresponding Author: acu.aad@ protonmail.com

${ }^{\text {B }}$ Self-employed Chinese Medicine Practitioner; NZ School of Acupuncture \& Traditional Chinese Medicine teacher/ supervisor

${ }^{\mathrm{C}}$ University of Western Australia

\begin{abstract}
Background: Pregnant women seek help for a range of physical and emotional pregnancy symptoms from traditional acupuncturists. Whether midwifery acupuncturists in Aotearoa New Zealand (Aotearoa NZ) provide acupuncture for antenatal anxiety and depression (AAD) in practice is currently unknown.
\end{abstract}

Aim: This qualitative arm of a mixed-methods study aimed to explore midwifery acupuncturist experiences of caring for pregnant women with AAD in Aotearoa NZ. The aim of the research was to examine the factors that influence midwifery acupuncturists' perceptions of acupuncture use for AAD.

Method: This was a mixed-methods study involving an online survey and in-depth interviews from a convenience sample of Aotearoa NZ midwifery acupuncturists who had completed a Certificate of Midwifery Acupuncture. This paper describes the thematic analysis of the semi-structured interviews.

Findings: The eight interviewees were mostly NZ European $(n=6)$ and Lead Maternity Carers (LMCs; $n=6$ ) caring for pregnant women in rural and urban locations throughout Aotearoa NZ. The interviews revealed an overarching theme, "helping midwives to navigate the ocean of AAD", which identified the lack of support participants experienced from current maternity health services for AAD in Aotearoa NZ. Subthemes "Missing the boat during pregnancy" expressed how participants felt no options were available for AAD prevention, while "keeping women afloat with no ship in sight" represented how interviewees tried to keep women in their care stable even though access to maternal mental health services was difficult. Participants felt acupuncture was a useful nonpharmaceutical tool that works; however, they had reservations about "adding acupuncture to the midwifery toolbox".

Conclusion: Aotearoa NZ midwifery acupuncturists were concerned about AAD and the limited conventional options available for women in their care. Acupuncture was viewed as a promising adjunct to usual treatment for AAD. Reservations included adding acupuncture to an already heavy midwifery workload, the cost of acupuncture, and the appropriateness of the treatment.

Keywords: acupuncture, antenatal, anxiety, depression, midwifery practice

\section{INTRODUCTION}

Anxiety and depression during pregnancy can cause adverse perinatal outcomes for both the mother and her baby (Eastwood et al., 2017; Grigoriadis et al., 2018), with an association found between antenatal anxiety (Grigoriadis et al., 2018) or antenatal depression (Underwood et al., 2016) and postnatal depression (PND). A considerable lifetime cost to individuals and the public sector for perinatal anxiety and depression in the United Kingdom has also been reported (Bauer et al., 2016). Within the context of this study, antenatal anxiety and depression (AAD) is defined as mild to moderate symptoms of anxiety and/or depression during pregnancy, which may or may not be clinically diagnosed.

Traditional acupuncture uses fine needles on specific points along body pathways to maintain balance in the body. This is in relation to traditional medicine theories such as those from China. Acupuncture has been found to be an effective intervention for antenatal depression in trials (Manber et al., 2009; Ormsby et al., 2020). This intervention may also offer potential relief for anxiety, stress, and comorbid physical symptoms for pregnant women with AAD. Acupuncture appears to be an acceptable treatment, as women with AAD have presented at an Aotearoa NZ hospital outpatient clinic for treatment by traditional acupuncturists (Betts, McMullan, \& Walker, 2016).

In Aotearoa NZ, some midwives practise acupuncture after completing a short midwifery acupuncture course (Betts, McMullan, \& Walker, 2016). These midwifery acupuncturists were taught limited acupuncture point strategies to support emotional wellbeing for pregnant women, along with basic pre- 
birth/labour treatments. However, it is currently unknown whether midwifery acupuncturists find acupuncture useful for AAD.

\section{BACKGROUND}

PND has been identified as a disorder that health resources in this country have been focused on, leaving antenatal mental health $(\mathrm{AMH})$ under-serviced (Signal et al., 2016). Although there has been a significant reduction in maternal suicide recorded between 2006 and 2016, suicide continues to be the leading cause of maternal mortality $(4.06 / 100,000$ new mothers; Perinatal and Maternal Mortality Review Committee, 2018). Interventions for AMH have been identified as being needed (Cornsweet Barber \& Starkey, 2015) and acupuncture may offer the potential to bridge this gap in conventional care.

Acupuncture has been found to be safe when administered by well-trained practitioners and there is evidence for its safety in major depressive disorders from a systematic review (McDonald \& Janz, 2017). Research on acupuncture specifically for antenatal depression is limited. However, one recent review found acupuncture may offer a referral pathway and reduce antenatal and postnatal depression (risk ratios 1.68, 95\% CI, 1.06 to 2.66, 1 trial; Smith et al., 2019). A more recent feasibility trial adds to this evidence, finding a significant reduction in the Edinburgh Postnatal Depression Scale ( $<<0.001$, mean difference [MD] 5.84, 95\% CI, -9.10 to -2.58$)$; the Depression, Anxiety and Stress Scale (-0.002, MD-4.83, 95\% CI, -7.65 to -2.01$)$; and the Kessler-6 ( $\mathrm{p}<0.001$; MD-6.42, 95\% CI, -10.05 to -2.79$)$ for those treated with acupuncture compared to usual treatment (Ormsby et al., 2020). The qualitative arms of this research reported that pregnant women described usual care as unsatisfactory, while they found acupuncture helpful for antenatal depression (Ormsby, Dahlen, $\&$ Smith, 2018). Further, maternity health professionals viewed acupuncture for antenatal depression as worth trying if it is safe (Ormsby, Dahlen, Ee, et al., 2018) and acupuncture was found to be an acceptable treatment for pregnant women with no reported adverse effects (Ormsby et al., 2020).

A systematic review of trials involving acupuncture use in general populations suggests a positive effect for anxiety, insomnia and depression (McDonald \& Janz, 2017). This perhaps also suggests the potential of acupuncture to alleviate these symptoms in pregnant women. A Cochrane review found that acupuncture may induce moderate reduction in depression severity compared to usual care/no treatment (standard mean deviation -0.66, 95\% CI -1.06 to $-0.25,5$ trials, 488 participants; Smith et al., 2018). One randomised controlled trial involving 572 participants reported reductions in the Patient Health Questionnaire (PHQ-9) depression scores for both acupuncture $(22.46,95 \%$ CI, 23.72 to 21.21) and counselling (21.73, 95\% CI, 23.00 to 20.45) when compared to usual care, finding the effect was sustained for six months (MacPherson et al., 2013). Further, analysis of these data found acupuncture also relieved symptoms like fatigue and insomnia (Hopton, MacPherson et al., 2014). This may be promising for pregnant women because physical symptoms such as insomnia (Emamian et al., 2019), fatigue (Qiu et al., 2012), and low back/pelvic pain (LBPP; Virgara et al., 2018) have been associated with perinatal depression and/or anxiety. Another Cochrane review (Liddle \& Pennick, 2015) also found that when acupuncture was used alongside usual care, there was a significant improvement of the LBPP during pregnancy when using the Disability Rating Index (DRI; median DRI 44 and 55 respectively, $\mathrm{p}=0.001)$. Both physical and emotional pregnancy symptoms (back pain, pelvic/hip pain, emotional problems and insomnia) have been treated by traditional acupuncturists in an Aotearoa NZ hospital maternity acupuncture clinic with high levels of satisfaction (Betts, McMullan, \& Walker, 2016).

Evidence-based referral pathways available to midwives for women with AAD in Aotearoa NZ are limited. Treatment of AAD symptoms may be an important preventative to avoid more serious $\mathrm{AMH}$ problems and acupuncture may offer an option in this area. Midwifery acupuncturists are well positioned to assess whether acupuncture might be a useful adjunct support for women with AAD. Their experiences of caring for women with AAD can inform other midwives, acupuncturists, pregnant women and healthcare providers on this topic.

\section{METHOD}

The qualitative aspect of this mixed methods study aimed to explore the experiences of midwifery acupuncturists in Aotearoa $\mathrm{NZ}$ who provided care to women with AAD. It involved a survey and semi-structured interviews. The findings from the survey will be reported at a later date. A constructivist pragmatic approach was applied for the study's qualitative arm. Exploring experiences can be useful for the complexity of health services research (Wisdom et al., 2012). A constructivist view implies participants construct reality from their experiences (Lee, 2012) which can allow the researcher to uncover consistencies and conflicts. Pragmatism suggests that consequence, context and circumstance can reveal the meaning of action and belief (Morgan, 2014). Thus, complexities of practice and pragmatism form the foundation for mixed-methods approaches (Long et al., 2018) that are relevant to examine complex interventions like traditional acupuncture and a midwife's experiences with this modality.

\section{Ethics}

Participants for the interviews were identified from the survey participants who provided names and contact details for interviews. Verbal consent was obtained before interviews were conducted. All data were de-identified and anonymised; email addresses, correspondence from surveys, and audio and transcription files from interviews were stored on password-protected devices with a two-step verification. Data and information will be kept securely for five years post-publication and then deleted.

Ethical approval was obtained from the New Zealand School of Acupuncture and Traditional Chinese Medicine Ethics Committee (NZSATCM/010).

\section{Recruitment}

Invitations to participate in an anonymous online survey (www. surveymonkey.com) were distributed by email to 172 midwives who had completed the Certificate of Midwifery Acupuncture between 2007 and 2019. A convenience sample of eight midwifery acupuncturists who met the criteria were interviewed. Inclusion criteria were that the participants were registered midwifery acupuncturists who worked with women during the antenatal period. English fluency with access to online services were also required. Interviews of 25-50 minutes duration were conducted in December 2019 and January 2020. Interviews were recorded via Skype (www.skype.com) or telephone, and then transcribed using Temi, an artificial intelligence online transcribing service (www. temi.com).

\section{Data collection and analysis}

Transcripts were de-identified and scrutinised for consistency with the recordings, and interviews were evaluated using thematic analysis (Castleberry \& Nolen, 2018) with a reflexive approach 
(Berger, 2015). Initial coding of data was undertaken and peer reviewed and themes developed based on the data.

Participants $(\mathrm{P})$ were provided with an interview number, and all quotes are identified by this number. An ellipsis indicates where words have been removed and [ ] indicates where wording has been added for clarity or to retain the anonymity of participants.

\section{FINDINGS}

\section{Participants}

Interviewees came from a range of locations, servicing urban $(n=3)$, rural $(n=2)$ or both urban and rural $(n=3)$ populations of pregnant women. They had varying levels of midwifery experience and were mostly NZ European ( $n=6)$ and/or Māori $(n=2)$, with six interviewees being LMCs and two being core midwives (Table 1).

\section{Table 1. Demographics of midwifery acupuncturist interviewees}

Demographic

Years practising midwifery

$<10$ years

$10-<20$ years

$20+$ years

Completed midwifery acupuncture course

In last 2 years

4

$2-<5$ years ago

2

$5+$ years ago

2

\section{Work type}

Lead Maternity Carer midwife

6

Core midwife

2

\section{Area type}

Urban

Rural

3

Both urban and rural

Age

25-44

45-54

3

55-64

3

2

\section{Ethnicity*}

NZ European/Pākehā

Māori

6

Other

*Multiple ethnicities possible

\section{Helping midwives to navigate the ocean of AAD}

An overarching theme from the eight participant interviews emerged as "helping midwives navigate the ocean of AAD". This theme identifies the lack of support these midwives felt existed within the current healthcare system for women with AAD, and also the lack of available treatment options, with acupuncture considered a viable additional treatment option. This overarching theme was derived from three themes, the first being "missing the boat during pregnancy" in which the participants suggested that treatment options were unavailable until women's mental health became extremely unstable. The second theme, "keeping pregnant women afloat with no ship in sight" reflects the situation where, although midwives did what they could to support women with mild to severe AAD, they sometimes had no options available from within the public health services, as illustrated by the phrase "no ship in sight". Finally, the theme of "adding acupuncture to the midwifery toolbox" identified that the participants felt integration of acupuncture is a potential "help" (a treatment option) for midwives when caring for women with AAD. Hence, the overarching theme captures the sense of acupuncture being an adjunct treatment option to inadequate mental health $(\mathrm{MH})$ services and which might be of help to midwives. Several subthemes were also present (Table 2). I would love to see DHBs [district health boards] employ an acupuncturist that works in the maternity unit. Wouldn't that be wonderful? And so, the women didn't pay for it (P5).

\section{Table 2. Themes arising from interviewees' experiences of working with} antenatal anxiety and depression

Overarching theme: Helping midwives navigate the ocean of AAD

Theme

Subthemes

Missing the boat during pregnancy Pregnant women feeling adrift Warning bells

Watching women deteriorate

Keeping pregnant women afloat It's a big concern

with no ship in sight

Strained resources

More options needed

Where's our help?

Building our own raft

Adding acupuncture to the Acupuncture really helps

midwifery toolbox

Considerations are required

Knowing the true story for $A A D$

Education

For acupuncturists

For pregnant women

\section{Missing the boat during pregnancy}

The participants identified "warning bells" which they described as being alerts for AAD. These included fatigue, crying, not being able to switch off, aches and pains, traumatic previous birth experiences, unrealistic expectations, hyperemesis, repeat admissions/presentations, anxiety, exhaustion and sleeplessness. Pregnancy progression was identified as potentially aggravating and escalating some women's mental instability, as identified by participant 8: anxiety... sleeplessness and things... really ramping up (P8). The sub-theme "pregnant women feeling adrift" described women feeling as though they are not being taken seriously by partners, family and/or healthcare professionals, leaving them not knowing where to turn (P3) and/or feeling judged (P4).

The sub-theme "watching women deteriorate" illustrated the helplessness some interviewees felt, due to there not being adequate services available for women with mild to moderate AAD. One participant described:

[a woman with nausea and] quite mild anxiety [who] phoned saying... she was concerned and... recognised she was probably more concerned than she needed to be. [After referring her to a social worker and general practitioner (GP) to no effect, this] escalated in...two weeks, [getting to the point where] the woman wasn't answering the phone... she was...crying and demanding an abortion because she had decided the baby was trying to kill her. (P3).

The midwifery participants described having to learn as they go: It was like a domino effect... when you look back it could have been almost like we missed the boat in pregnancy (P1). 


\section{Keeping pregnant women afloat with no ship in sight}

The theme "keeping pregnant women afloat with no ship in sight" highlighted services and options for AAD as being unsatisfactory, leaving midwives feeling overloaded, unsupported and asking "Where's our help?" If we nip it in the bud earlier in the pregnancy... it doesn't escalate (P3).

Participants identified that healthcare resources were "stretched"; however, this didn't mitigate the sense of frustration felt. For nonacute cases of AAD, LMCs are required to refer to GPs (Ministry of Health, 2012) who can then refer women to limited free counselling if the GP agrees with the diagnosis. The participants described this pathway as problematic and "strained", with services being overrun: The last two referrals I've done for anxiety or depression, those women have been put on a waiting list (P3).

Participant 3 resorted to a convoluted pathway by getting women to ask their GP for referral to maternal mental health (MMH) services because referrals from midwives were not being accepted.

Another participant had no MMH services available in her area, stating if they're not acute... it can be a six-week lead in time (P5). Acute cases also experienced wait times: We were going to refer [to] emergency psych services, and they said there's about an eight hour wait (P5).

Participants identified "more options needed" because women were often reluctant to follow the conventional option because they had been down that road before and [were] not that interested (P8) or They just keep saying 'no, no, no' or 'I've tried that before, it won't work' (P5).

Participants explained that women would follow through for physical things [but] when it comes to... anxiety and depression and MH issues, that's when they're a bit more reluctant...they're...like, oh yeah, okay. But then not do it (P8).

One interviewee described her scope of practice as normal pregnancies (P8), yet participants found themselves often learning as [they] go and feeling they almost had to treat women for AAD (P1).

It's so dumb... we do the screening, but we can't do anything with the screening... we don't have any options (P6).

Participant 8 stated I need to hand her over, but the people that I need to really hand her over to, are just not actually available...on paper they are. But in reality, it's just lacking... it takes its toll (P8).

One interviewee thought the lack of options was why midwives get a bit overloaded (P5), while another described a colleague who found it exhausting having to do the needles all the time, stating we're helping women, but where's our help? (P6).

Although most participants identified that more support was necessary, one experienced participant with an interest in $\mathrm{MH}$ stated that, if [women are] better supported antenatally then potentially they'll have a better birth, so then they'll recover better (P7). She felt that acupuncture was one way she could address this, although she did not believe acupuncture to be the only answer. It was believed the more women were referred to various modalities, the better they do (P7). She suggested to let them have... whatever they need... the sooner the better (P7). This core midwife made use of various free complementary and alternative medicine (CAM) services from a traditional acupuncture clinic, a hospital and a marae, along with the acupuncture she was able to administer herself, with there being no cost to women. She felt it was all quite good really (P7).

\section{Adding acupuncture to the midwifery toolbox}

The theme "adding acupuncture to the midwifery toolbox" was identified by participants who felt positive about the effects of acupuncture as a treatment option for AAD.

The participants felt "acupuncture really helps": I don't think I've ever had any negatives about acupuncture (P2); It actually does work (P6); It's really been such a useful tool (P8); Even though I don't practise [acupuncture anymore] I do refer (P1).

Participants stated that acupuncture for AAD would be amazing... especially if [women] can access it for free (P4), and for those that want to avoid medication (P8).

One participant identified that a woman's wellbeing and outlook were quite uplifted. [She] actually sought help... doing...those needles, it's actually changed their mindset (P6). While discussing another case where a first baby was very preterm [and the woman became] quite agitated at the point where that baby had been born, this same participant described how points for calming, anxiety and sleep were used, which really relaxed the woman, so she slept really well afterwards (P6).

"Considerations are required" summed up the reservations midwifery acupuncturists held about acupuncture for pregnant women. These included the financial barrier of accessing care due to the cost of acupuncturists in the private sector, as well as considering the different types of acupuncturists and whether they have experience of treating pregnant women.

Interviewees thought it would be good to have a funded acupuncture clinic...so it's no cost for [women] but we're not out of pocket either (P6).

One participant also felt by having acupuncturists working in hospitals it would reduce the woman's hospital stay [for conditions like] hyperemesis (P5).

Participants thought it important that acupuncturists know the medical history of women with AAD from midwifery colleagues, rather than relying on women to pass on information. This sharing of information was also thought important in the case of referrals for induction and high-risk pregnancies.

In addition, participants felt that their midwifery colleagues needed to be better educated about acupuncture and what it can do, with most knowing about its effects for getting ready for birth or turning babies... but not really for the emotional stuff (P8). Participants identified that most pregnant women have never had acupuncture before or considered it as a treatment option (P3) and some participants had never thought to use acupuncture for AAD. Acupuncture was described as a new thing for people to get their heads around, [and educating people] that that's an option is needed (P2).

Aversion to needles was described as a potential barrier to acupuncture; however, participants explained that it was helpful showing women how unlike acupuncture needles are to needles that take blood or give injections (P3). One participant started with just one needle, and commented that then women realise it doesn't actually hurt (P8). Another interviewee described the absolute needle phobia and anxiety of one woman who was not keen to use [acupuncture] for pre-birth but when she didn't go into labour using acupressure, acupuncture was then acceptable, and it worked a treat. If a need...comes up... they will actually just... do it (P4).

\section{DISCUSSION}

This study set out to explore the factors that influence midwifery acupuncturists' perceptions of acupuncture use for AAD. The 
participants have identified that there is little existing support for women with mental health issues and that providing acupuncture may be an additional tool of support. They described acupuncture for $\mathrm{AAD}$ as a novel approach which would require education to support its adaptation into practice, even though women have been found to view acupuncture as acceptable and satisfactory (Betts, McMullan, \& Walker, 2016; Williams et al., 2020). Positive perceptions of acupuncture by midwives have been reported previously and include the perception that acupuncture had potential as a preventative to medicalised treatment (Ormsby, Dahlen, Ee, et al., 2018).

The participants identified that women's mental health appeared to deteriorate during pregnancy. Evidence suggests that this may be the case, with the prevalence of self-reported anxiety in the first trimester from 102 studies in 34 countries estimated as $18.2 \%$ (95\% CI, 13.6-22.8, 10 studies, $\mathrm{n}=10,577)$ compared to $24.6 \%$ (95\% CI, 21.2-28.0, 33 studies, $\mathrm{n}=116,720$ ) in the third trimester (Dennis et al., 2017). A randomised controlled trial found that acupuncture was as useful as counselling, with both interventions significantly reducing depression compared to usual care (MacPherson et al., 2013). This trial also found fatigue and insomnia were relieved (Hopton, MacPherson et al., 2014).

Participants in this study saw a need for other options and identified that some women will refuse conventional treatments because they have not worked in the past; this aligns with the findings of Ormsby, Dahlen, and Smith (2018). National and international studies have found that some women with AAD are less likely to be honest when consulting with, or perceive risk differently to, medical professionals (Cornsweet Barber et al., 2017; Forder et al., 2019). This may result in "at risk" women not being identified, making prevention and treatment of comorbid physical symptoms an important part of assessment for AAD. Participants described pregnant women as readily exploring treatments for physical symptoms yet being less likely to follow through with conventional options should they feel mentally unwell. This study emphasises the need for further research into preventative interventions for AAD for pregnant women in Aotearoa NZ. Participants expressed a desire for more support in caring for women with AAD. They viewed acupuncture as a beneficial treatment option; however, affordability of the treatment by acupuncturists who practise privately was a major concern.

Acupuncture may be perceived as relating more to treatment for physical symptoms in Aotearoa NZ, due to its inclusion in the treatment of injuries that are subsidised by public health insurance (Accident Compensation Corporation, n.d.). Having this increased acceptance, it may offer another option for AAD, with potential for promoting "passive recipients" to "active participants" (Hopton, Eldred, \& MacPherson, 2014). One participant described how when acupuncture points were used for emotional wellbeing, they helped change a woman's outlook, enabling her to seek help. Previous studies have identified acupuncture as supporting empowerment, optimism (Betts, Smith, \& Dahlen, 2016), motivation (Schnyer et al., 2003), and physical and emotional wellbeing (Smith et al., 2002) for pregnant women.

Physical symptoms such as sleep problems, fatigue and pain were termed by the participants as "warning bells" and were viewed as potentially amplifying $\mathrm{AAD}$, a finding that has also identified by Emamian et al. (2019). Early treatment options for these symptoms are worthy of further investigation to prevent potential mental health deterioration. More research is needed to identify whether providing acupuncture for these early physical pregnancy symptoms has a potential to influence AAD.

A lack of referral options and the desire for education on AAD were emphasised by the participants. Making options, such as acupuncture, available as preventative strategies may help to spread the work overload participants felt when caring for women with AAD.

Most midwifery acupuncturists know to refer to traditional acupuncturists for more complex problems, as has been found in other CAM research (Hall et al., 2013). Closer collaboration between midwives and acupuncturists was thought important for acupuncture use in $\mathrm{AAD}$, as well as in situations involving induction and high-risk pregnancies.

This research highlights that, for midwives, pregnant women and the wider community, education about acupuncture and the evidence that supports its use are needed. Acupuncture was perceived as a useful preventative treatment option for AAD by the midwifery participants, with the potential to change women's outlook, enabling them to proactively seek help.

There is promising evidence for acupuncture as a preventative treatment for AAD as well as for physical symptoms of concern, such as insomnia, fatigue, pain, nausea and vomiting, and the stress for some around pregnancy progression. Interviewees in this study expressed support for adding acupuncture to their "toolkit" for AAD. Exploring ways of establishing easy referral pathways that could minimise extra work burden for midwives will be required.

\section{STRENGTHS AND LIMITATIONS}

This research has been the first to explore Aotearoa NZ midwifery acupuncturists' experiences of caring for women with AAD. Participants were from a range of regions, servicing both urban and rural populations. This is a strength and allows a range of views to emerge from midwifery acupuncturists caring for women with AAD, who encounter or interact with various regional rural and metropolitan MMH services. This sample may not represent midwifery acupuncturists and the results cannot be generalised to others. Aotearoa NZ midwifery acupuncturists may have differing views of acupuncture to those of the general midwifery population in Aotearoa NZ and overseas. It would be interesting to make comparisons between these groups in future research.

Twelve to sixteen interviews are thought adequate to reach code saturation, with as few as six revealing basic main themes (Hennink et al., 2017). However, only eight interviews were achieved, which is a limitation. Several participants found Skype access burdensome, having to download an app they did not normally use. As a result, the majority of interviews were conducted by telephone, which limits the researchers' ability to observe visual cues. Main meta-themes were obvious within the eight interviews and the last interview yielded no new information.

\section{CONCLUSION}

Midwifery acupuncturists in this study expressed concern about the limited services available for pregnant women displaying mild to moderate, and sometimes severe, AAD. They discussed how women currently have few options for AAD treatment and that acupuncture may be an acceptable and beneficial option for AAD prevention; but further research is needed.

With conventional treatment for AAD sometimes being found unsatisfactory, difficult to access or unacceptable, acupuncture may provide a physical, non-pharmaceutical alternative. Participants described acupuncture as a useful tool, and having the potential to change the mindsets of women with AAD. Some participants desired to see acupuncture funded publicly so there was no cost to women. Greater collaboration between midwives and acupuncturists may be required, bearing in mind Privacy Act obligations (Ministry of Justice, 2020), to ensure acupuncturists have the correct and relevant information about women for AAD treatment. Education about acupuncture and acupuncturists is 
needed for pregnant women and midwives, as acupuncture was not well known as an option for AAD. By using acupuncture, participants felt they were helping women; however, this was viewed by some as adding to their already stretched workload.

\section{ACKNOWLEDGEMENTS AND CONFLICT OF INTEREST}

A special thanks to all the midwifery acupuncturists who found the time to take part in this research. Debra Betts taught the Certificate of Midwifery Acupuncture and funding was provided by the New Zealand School of Acupuncture and Traditional Chinese Medicine, which granted full scholarship for Leeana Lowe to undertake a MHS (Chinese Medicine) at the same insititution.

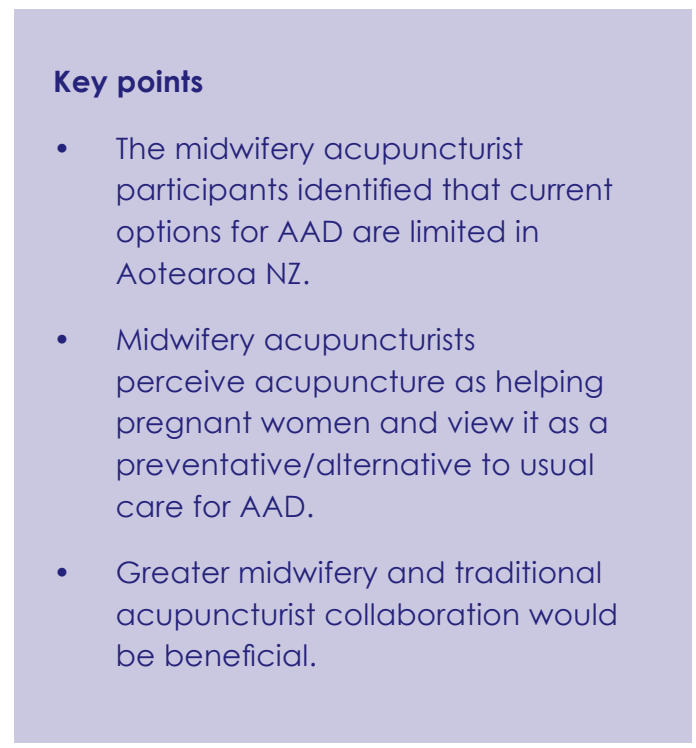

\section{REFERENCES}

Accident Compensation Corporation (ACC) - Te Kaporeihana Āwhina Hunga Whara. (2020, August 7). Treatment we can help pay for. https:// www.acc.co.nz/im-injured/what-we-cover/treatment-we-pay-for/ Bauer, A., Knapp, M., \& Parsonage, M. (2016). Lifetime costs of perinatal anxiety and depression. Journal of Affective Disorders, 192, 8390. https://doi.org/10.1016/j.jad.2015.12.005

Berger, R. (2015). Now I see it, now I don't: researcher's position and reflexivity in qualitative research. Qualitative Research, 15(2), 219-234. https://doi.org/10.1177/1468794112468475

Betts, D., McMullan, J., \& Walker, L. (2016). The use of maternity acupuncture within a New Zealand public hospital: Integration within an outpatient clinic. New Zealand College of Midwives Journal, 52, 45-49. https://doi.org/10.12784/nzcomjnl52.2016.7.45-49

Betts, D., Smith, C. A., \& Dahlen, H. G. (2016). Does acupuncture have a role in the treatment of threatened miscarriage? Findings from a feasibility randomised trial and semi-structured participant interviews. BMC Pregnancy and Childbirth, 16(1), article 298. https://doi. org/10.1186/s12884-016-1092-8

Castleberry, A., \& Nolen, A. (2018). Thematic analysis of qualitative research data: Is it as easy as it sounds? Currents in Pharmacy Teaching and Learning, 10(6), 807-815. https://doi.org/10.1016/j. cptl.2018.03.019

Cornsweet Barber, C., Panettierre, M., \& Starkey, N. J. (2017). How am I, really? Perceptions of health and distress by women and their midwives. Journal of Reproductive and Infant Psychology, 35(3), 261-272. https://doi.org/10.1080/02646838.2017.1310375

Cornsweet Barber, C., \& Starkey, N. J. (2015). Predictors of anxiety among pregnant New Zealand women hospitalised for complications and a community comparison group. Midwifery, 31(9), 888-896. https:// doi.org/10.1016/j.midw.2015.04.017

Dennis, C. L., Falah-Hassani, K., \& Shiri, R. (2017). Prevalence of antenatal and postnatal anxiety: Systematic review and meta-analysis. British Journal of Psychiatry, 210(5), 315-323. https://doi.org/10.1192/ bjp.bp.116.187179
Eastwood, J., Ogbo, F. A., Hendry, A., Noble, J., \& Page, A. (2017).

The impact of antenatal depression on perinatal outcomes in Australian women. PLoS ONE, 12(1), e0169907. https://doi.org/10.1371/journal. pone.0169907

Emamian, F., Khazaie, H., Okun, M. L., Tahmasian, M., \& Sepehry, A. A. (2019). Link between insomnia and perinatal depressive symptoms: A meta-analysis. Journal of Sleep Research, 28(6), e12858. https://doi. org/10.1111/jsr.12858

Forder, P. M., Rich, J., Harris, S., Chojenta, C., Reilly, N., Austin, M. P., \& Loxton, D. (2019). Honesty and comfort levels in mothers when screened for perinatal depression and anxiety. Women and Birth, 3(2), e142-e150. https://doi.org/10.1016/j.wombi.2019.04.001

Grigoriadis, S., Graves, L., Peer, M., Mamisashvili, L., Tomlinson, G., Vigod, S. N., Dennis, C.-L., Steiner, M., Brown, C., Cheung, A., Dawson, H., Rector, N. A., Guenette, M., \& Richter, M. (2018). A systematic review and meta-analysis of the effects of antenatal anxiety on postpartum outcomes. Archives of Women's Mental Health, 22(5), 543556. https://doi.org/10.1007/s00737-018-0930-2

Hall, H. G., McKenna, L. G., \& Griffiths, D. L. (2013). From alternative, to complementary to integrative medicine: Supporting Australian midwives in an increasingly pluralistic maternity environment. Women and Birth, 26(2), e90-e93. https://doi.org/10.1016/j. wombi.2012.11.004

Hennink, M. M., Kaiser, B. N., \& Marconi, V. C. (2017). Code saturation versus meaning saturation: how many interviews are enough? Qualitative Health Research, 27(4), 591-608. https://doi. org/10.1177/1049732316665344

Hopton, A., Eldred, J., \& MacPherson, H. (2014). Patients' experiences of acupuncture and counselling for depression and comorbid pain: a qualitative study nested within a randomised controlled trial. BMJ Open, 4(6), e005144. https://doi.org/10.1136/bmjopen-2014-005144

Hopton, A, MacPherson, H., Keding, A., \& Morley, S. (2014). Acupuncture, counselling or usual care for depression and comorbid pain: secondary analysis of a randomised controlled trial. BMJ Open, 4(5), e004964. https://doi.org/10.1136/bmjopen-2014-004964

Lee, C. G. (2012). Reconsidering constructivism in qualitative research. Educational Philosophy and Theory, 44(4), 403-412. https://doi. org/10.1111/j.1469-5812.2010.00720.x

Liddle, S., \& Pennick, V. (2015). Interventions for preventing and treating low-back and pelvic pain during pregnancy. Cochrane Database of Systematic Reviews, 9, CD001139. https://doi.org/10.1002/14651858. CD001139.pub4.

Long, K. M., McDermott, F., \& Meadows, G. N. (2018). Being pragmatic about healthcare complexity: our experiences applying complexity theory and pragmatism to health services research. $B M C$ Medicine, 16, article 94. https://doi.org/10.1186/s12916-018-1087-6 MacPherson, H., Richmond, S., Bland, M., Brealey, S., Gabe, R., Hopton, A., Keding, A., Lansdown, H., Perren, S., Sculpher, M., Spackman, E., Torgerson, D., \& Watt, I. (2013). Acupuncture and counselling for depression in primary care: A randomised controlled trial. PLoS Medicine, 10(9), e1001518. https://doi.org/10.1371/journal. pmed.1001518

Manber, R., Schnyer, R. N., Lyell, D., Chambers, A. S., Caughey, A. B., Druzin, M., Carlyle, E., Celio, C., Gress, J., Huang, M., Kalista, T., Martin-Okada, R., \& Allen, J. J. B. (2009). Acupuncture for depression during pregnancy. A randomized controlled trial. American Journal of Obstetrics and Gynecology, 201(6 SUPPL. 1), S19. https://doi. org/10.1097/AOG.0b013e3181cc0816

McDonald, J., \& Janz, S. (2017). The acupuncture evidence project: A comparative literature review. Australian Acupuncture and Chinese Medicine Association Ltd. https://acupunctuur-in-je-element.nl/ wp-content/uploads/2019/01/The_Acupuncture_Evidence_Project_ McDonald-and-Janz_2017.pdf

Ministry of Health - Manatu Hauora. (2012). Guidelines for Consultation with Obstetric and Related Medical Services (Referral Guidelines). https://www. health.govt.nz/system/files/documents/publications/referral-glines-jan12.pdf Ministry of Justice - Te Tāhūo te Ture. (2020). Privacy Act 2020, No 31 (as at 01 April 2021). New Zealand Legislation Paliamentary Counsel Office - Te Tari Tohutohu Pāremata. https://www.legislation.govt.nz/ act/public/2020/0031/latest/LMS23223.html

Morgan, D. L. (2014). Pragmatism as a paradigm for social research. Qualitative Inquiry, 20(8), article 10451053. https://doi. org/10.1177/1077800413513733

Ormsby, S. M., Dahlen, H. G., Ee, C. C., Keedle, H., \& Smith, C. A. (2018). 'Acupuncture for antenatal depression: It's worth giving it a go' - A qualitative study. Women and Birth, 31(3), 166-176. https://doi. org/10.1016/j.wombi.2017.09.018 
Ormsby, S. M., Dahlen, H. G., \& Smith, C. A. (2018). Women's experiences of having depression during pregnancy and receiving acupuncture treatment - A qualitative study. Women and Birth, 31(6), 469-478. https://doi.org/10.1016/j.wombi.2017.10.009

Ormsby, S. M., Smith, C. A., Dahlen, H. G., \& Hay, P. J. (2020).

The feasibility of acupuncture as an adjunct intervention for antenatal depression: a pragmatic randomised controlled trial. Journal of Affective Disorders, 275, 82-93. https://doi.org/10.1016/j.jad.2020.05.089

Perinatal and Maternal Mortality Review Committee. (2018). Twelfth Annual Report of the Perinatal and Maternal Mortality Review Committee: Reporting mortality and morbidity 2016. Eighth report to the Health Quality \& Safety Commission, New Zealand. http://www.hqsc.govt.nz/ our-programmes/mrc/pmmrc

Qiu, C., Gelaye, B., Fida, N., \& Williams, M. A. (2012). Short sleep duration, complaints of vital exhaustion and perceived stress are prevalent among pregnant women with mood and anxiety disorders. BMC Pregnancy and Childbirth, 12, article 104. https://doi. org/10.1186/1471-2393-12-104

Schnyer, R. N., Manber, R., \& Fitzcharles, A. J. (2003). Acupuncture treatment for depression during pregnancy: Conceptual framework and two case reports. Complementary Health Practice Review, 8(1), 40-53. https://doi.org/10.1177/1076167502238384

Signal, T. L., Paine, S. J., Sweeney, B., Muller, D., Priston, M., Lee, K., Gander, P., \& Huthwaite, M. (2016). The prevalence of symptoms of depression and anxiety, and the level of life stress and worry in New Zealand Māori and non-Māori women in late pregnancy. Australian and New Zealand Journal of Psychiatry, 51(2), 168-176. https://doi. org/10.1177/0004867415622406

Smith, C., Armour, M., Lee, M. S., Wang, L.-Q., \& Hay, P. J. (2018). Acupuncture for depression (Review). Cochrane Database of Systematic Reviews, 3, CD004046. https://doi.org/10.1002/14651858.CD004046. pub4

Smith, C., Crowther, C., \& Beilby, J. (2002). Acupuncture to treat nausea and vomiting in early pregnancy: A randomized controlled trial. Birth, 29(1), 1-9. https://doi.org/10.1046/j.1523-536x.2002.00149.x

Smith, C., Shewamene, Z., Galbally, M., Schmied, V., \& Dahlen, H. (2019). The effect of complementary medicines and therapies on maternal anxiety and depression in pregnancy: A systematic review and meta-analysis. Journal of Affective Disorders, 245, 428-439. https://doi. org/10.1016/j.jad.2018.11.054

Underwood, L., Waldie, K., D’Souza, S., Peterson, E. R., \& Morton, S. (2016). A review of longitudinal studies on antenatal and postnatal depression. Archives of Women's Mental Health, 19(5), 711-720. https:// doi.org/10.1007/s00737-016-0629-1

Virgara, R., Maher, C., \& Van Kessel, G. (2018). The comorbidity of low back pelvic pain and risk of depression and anxiety in pregnancy in primiparous women. BMC Pregnancy and Childbirth, 18, article 288. https://doi.org/10.1186/s12884-018-1929-4

Williams, H., Sweet, L., \& Graham, K. (2020). Acupuncture during pregnancy and the perinatal period: Women's attitudes, beliefs and practices. Women and Birth, 33(3), e286-e294. https://doi.org/10.1016/j. wombi.2019.04.010

Wisdom, J. P., Cavaleri, M. A., Onwuegbuzie, A. J., \& Green, C. A. (2012). Methodological reporting in qualitative, quantitative, and mixed methods health services research articles health services research. Health Services Research, 42(2), 721-745. https://doi.org/10.1111/j.14756773.2011.01344.x

\section{Accepted for Publication April 2021}

Lowe, L-A., \& Betts, D. (2021). Midwives' experiences of caring for women with antenatal anxiety and depression: A qualitative study with midwifery acupuncturists. New Zealand College of Midwives Journal, 57, 20-26.

https://doi.org/10.12784/nzcomjnl57.2021.3.20-26 